\title{
Feasibility of Monitoring Health and Well-being in Emerging Adults: Pilot Longitudinal Cohort Study
}

Reidar P Lystad ${ }^{1}$, PhD; Diana Fajardo Pulido ${ }^{1}$, MIPH/MHM; Lorna Peters ${ }^{2}$, PhD; Melissa Johnstone ${ }^{3}, \mathrm{PhD}$; Louise A Ellis ${ }^{1}, \mathrm{PhD}$; Jeffrey Braithwaite ${ }^{1}, \mathrm{PhD}$; Viviana Wuthrich ${ }^{2}, \mathrm{PhD}$; Janaki Amin ${ }^{4}, \mathrm{PhD}$; Cate M Cameron ${ }^{5,6}, \mathrm{PhD}$; Rebecca J Mitchell ${ }^{1}, \mathrm{PhD}$

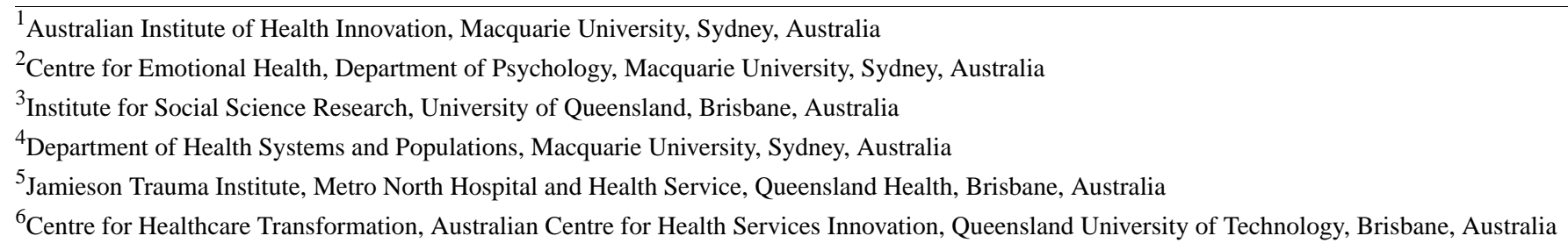

\section{Corresponding Author:}

Reidar P Lystad, PhD

Australian Institute of Health Innovation

Macquarie University

75 Talavera Road

Sydney, 2109

Australia

Phone: 61298502464

Email: reidar.lystad@mq.edu.au

\section{Abstract}

Background: Emerging adulthood is a distinct segment of an individual's life course. The defining features of this transitional period include identity exploration, instability, future possibilities, self-focus, and feeling in-between, all of which are thought to affect quality of life, health, and well-being. A longitudinal cohort study with a comprehensive set of measures would be a valuable resource for improving the understanding of the multifaceted elements and unique challenges that contribute to the health and well-being of emerging adults.

Objective: The main aim of this pilot study was to evaluate the feasibility and acceptability of recruiting university graduates to establish a longitudinal cohort study to inform the understanding of emerging adulthood.

Methods: This pilot study was conducted among graduates at a large university. It involved collecting web-based survey data at baseline (ie, graduation) and 12 months post baseline, and linking survey responses to health records from administrative data collections. The feasibility outcome measures of interest included the recruitment rate, response rate, retention rate, data linkage opt-out rate, and availability of linked health records. Descriptive statistics were used to evaluate the representativeness of the sample, completeness of the survey responses, and data linkage characteristics.

Results: Only 2.8\% of invited graduates (238/8532) agreed to participate in this pilot cohort study, of whom 59.7\% (142/238) responded to the baseline survey. The retention rate between the baseline and follow-up surveys was $69.7 \%$ (99/142). The completeness of the surveys was excellent, with the proportion of answered questions in each survey domain ranging from $87.3 \%$ to $100 \%$ in both the baseline and follow-up surveys. The data linkage opt-out rate was $32.4 \%$ (77/238).

Conclusions: The overall recruitment rate was poor, while the completeness of survey responses among respondents ranged from good to excellent. There was reasonable acceptability for conducting data linkage of health records from administrative data collections and survey responses. This pilot study offers insights and recommendations for future research aiming to establish a longitudinal cohort study to investigate health and well-being in emerging adults.

Trial Registration: Australian New Zealand Clinical Trials Registry number ACTRN12618001364268; https://tinyurl.com/teec8wh

International Registered Report Identifier (IRRID): RR2-10.2196/16108 
(JMIR Form Res 2022;6(1):e30027) doi: 10.2196/30027

\section{KEYWORDS}

young adult; emerging adulthood; health; well-being; health-related quality of life; feasibility; monitoring; pilot study; longitudinal; cohort; youth; acceptability; survey; quality of life

\section{Introduction}

Emerging adulthood is the life stage between adolescence and young adulthood, lasting approximately from ages 18 to 25 years $[1,2]$. There are many events and factors that can impact the life course of emerging adults [1,2]. Changing health states, different lived experiences, exposure to diverse opportunities, education, and influences from cultural and socioeconomic circumstances can challenge the transition from late adolescence to adulthood, and they have a significant impact on the health and well-being of emerging adults [2-5].

Few longitudinal studies have investigated aspects of health-related quality of life (HRQoL) and well-being in emerging adults. In the United States, 2 university cohorts were established at Harvard University to investigate risk factors for chronic diseases and long-term health in nurses and health professionals [6,7]. In Spain, a prospective university graduate cohort was established at Seguimiento Universidad de Navarra (SUN) to examine dietary habits in the Mediterranean region $[8,9]$. The scope of the SUN study was subsequently broadened to examine other risk factors and health conditions and expanded to include graduates from 5 other Spanish universities [9]. No longitudinal cohort study comprehensively investigating HRQoL and well-being in emerging adults has been undertaken in Australia. In Australia, Eisenberg et al [10] examined phase transitions of emerging adults, but did not report on any HRQoL or well-being measures. Landstedt et al [11] investigated mental health in a cohort of young Australian adults, but the participants were only asked a single question (ie, "How healthy have you felt mentally during the past 12 months?"), which was not included at baseline.

Conducting a large, prospective longitudinal cohort study of emerging adults using a comprehensive set of measures (eg, physical and mental health, risk factors, life events, resilience, education and employment factors, and social connectedness) would be a unique and valuable resource for improving our understanding of the determinants of healthy and resilient individuals in our society. However, because large, prospective longitudinal cohort studies can be very costly and resource-consuming undertakings, it is essential to first determine the feasibility of conducting such studies.

This pilot study, therefore, aimed to establish the feasibility of recruiting university graduates to establish a large, prospective longitudinal cohort study to inform our understanding of emerging adulthood. Specifically, this pilot study evaluated the following: (1) the feasibility of research methods to recruit university graduates at a large Australian university, including determination of the opt-out rate for data linkage of health records and survey responses; (2) the representativeness of the recruited participants; (3) the ability to obtain baseline survey data, including completion of individual survey instruments;
(4) the ability to retain participants and collect follow-up survey data 12 months post baseline, including the completion of individual survey instruments; and (5) opportunities for improving the design of future studies.

\section{Methods}

\section{Registration}

This study was registered with the Australian New Zealand Clinical Trials Registry (ACTRN) on August 14, 2018 (ACTRN12618001364268). The study protocol was published on April 23, 2020 (international registered report identifier: DERR1-10.2196/16108) [12].

\section{Study Design}

This pilot longitudinal cohort study was conducted at Macquarie University in Sydney, Australia. It involved collecting information via web-based surveys (ie, at baseline and 12 months post baseline) and health data record linkage.

\section{Recruitment}

All students graduating from Macquarie University in 2018 $(\mathrm{N}=8532)$ were eligible to participate in this study. Macquarie University is a large public university located in a suburban area of Sydney, Australia. At the time of participant recruitment, the university comprised five faculties (ie, Faculty of Arts, Faculty of Business and Economics, Faculty of Human Sciences, Faculty of Medicine and Health Sciences, and Faculty of Science and Engineering), which collectively hosted approximately 45,000 students, including 33,000 undergraduate students, 9000 postgraduate students, and 1500 higher-degree research students.

The graduates were invited to participate via email during the autumn (ie, April) and spring (ie, September) graduation periods. Email invitations included a unique link to a purpose-made website where the graduates were informed about the study before consenting to participate. The initial invitation was followed by 3 reminder emails over a 6-week period. After completing the web-based registration form, participants received an email with an individualized link to the baseline survey.

An incentive to participate was introduced for the second (ie, September) graduation period. The incentive to participate was an entry into a random draw to win 1 of 3 prizes, namely an iPad mini (Apple Inc, first prize) or movie tickets (second and third prizes).

\section{Survey Data Collection}

Surveys were administered via the web-based Qualtrics XM platform (Qualtrics International Inc) at baseline and 12 months post baseline. The baseline and 12-month follow-up surveys comprised the same battery of validated questionnaires and instruments designed to capture data regarding 
sociodemographic factors, education, employment, job satisfaction, mentoring, self-perceived physical and mental health status, work-life balance, connectedness, resilience, injury, risk behaviors, and life events, as well as social media and technology use. For a detailed overview of the domains and specific questionnaires included in the web-based surveys, see the published study protocol [12].

\section{Health Record Linkage}

Survey responses were linked to personal health information from administrative data collections (ie, ambulance dispatches, emergency department presentations, hospital admissions, cancer registry, and mortality records) in New South Wales (NSW) from April 1, 2018, to 12 months after the completion of the baseline survey. Participants had an opportunity to opt out of having their survey responses linked to their health records during the web-based registration process. The secure health data linkage was conducted by the Centre for Health Record Linkage (CHeReL).

\section{Outcome Measures}

The specific feasibility outcome measures for this pilot study were as follows:

1. Recruitment rate: Calculated as the number of graduates who registered to participate in the pilot study, divided by the total number of graduates.

2. Response rate: Calculated as the number of registered participants who completed the baseline survey, divided by the total number of registered participants.

3. Representativeness: Evaluated by comparing the distribution of graduates and responding participants by faculty and level of qualification.

4. Retention rate: Calculated as the number of participants who completed both the baseline and 12-month follow-up surveys, divided by the number of participants who completed the baseline survey only.

5. Completeness: Calculated as the proportion of missing data for each survey item separately for the baseline and follow-up surveys. For the purpose of this study, survey completeness was categorized as poor $(<50 \%)$, average ( $50 \%$ to $<75 \%)$, good $(75 \%$ to $<95 \%)$, or excellent $(\geq 95 \%)$.

6. Data linkage opt-out rate: Calculated as the number of registered participants who opted out of having their survey responses linked to their health records, divided by the total number of registered participants.

7. Data linkage rate: Calculated as the number of participants who did not opt out of having their survey responses linked to their health records and had one or more health records identified in the Master Linkage Key, divided by the number of registered participants who did not opt out of having their survey responses linked to their health records.

8. Linked record availability: Calculated as the number of available linked health records in each administrative data collection.

\section{Data Analysis}

All data were analyzed using SAS, version 9.4 ( SAS Institute). The recruitment rate, response rate, retention rate, data linkage opt-out rate, data linkage rate, and linked record availability rate were calculated as described above and presented as proportions. Descriptive statistics were used to evaluate the representativeness of the sample and completeness of the baseline and follow-up surveys.

\section{Results}

A total of 8532 graduates were invited to participate in this pilot study, of whom 238 agreed to participate (Figure 1). This equated to a recruitment rate of $2.8 \%$. Of the 238 graduates who registered to participate in this study, 142 answered the baseline survey. This equated to a response rate of $59.7 \%$. The majority of respondents were female $(100 / 142,70.4 \%)$, single $(79 / 142$, $55.6 \%$ ), and born in Australia (95/142, 66.9\%) (Table 1). Compared to the invited graduates, the sample of responding participants was significantly different in its distribution by gender $(P<.001)$, but not by level of award $(P=.14)$ or graduation time $(P=.08)$ (Table 2$)$.

Figure 1. Flow diagram of the selection of the study participants.

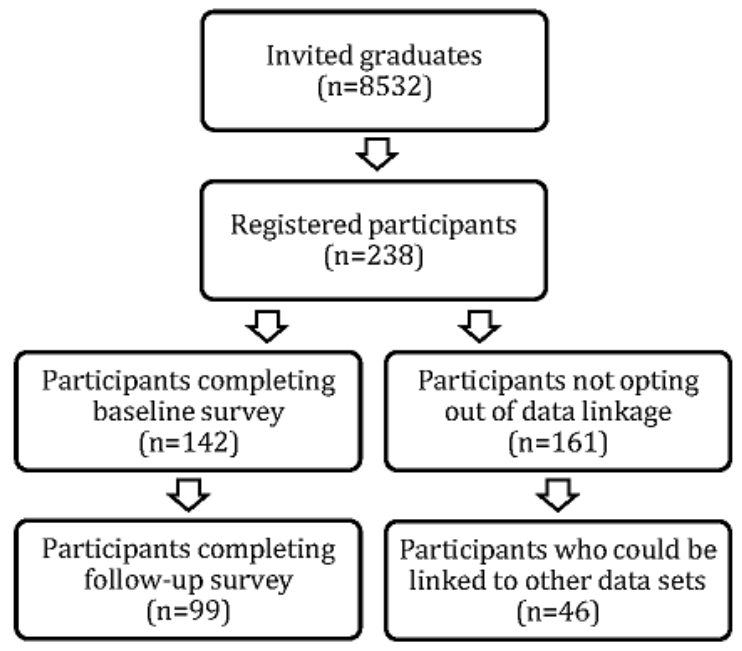


Table 1. Demographic characteristics of responding participants.

\begin{tabular}{|c|c|}
\hline Characteristic & Respondents (n=142), n (\%) \\
\hline \multicolumn{2}{|l|}{ Gender } \\
\hline Female & $100(70.4)$ \\
\hline Male & $41(28.9)$ \\
\hline Other or unspecified & $1(0.7)$ \\
\hline \multicolumn{2}{|l|}{ Marital status } \\
\hline Single & $79(55.6)$ \\
\hline In relationship, not living with partner & $5(3.5)$ \\
\hline In relationship, living with partner & $24(16.9)$ \\
\hline Married & $32(22.5)$ \\
\hline Divorced & $2(1.4)$ \\
\hline \multicolumn{2}{|l|}{ Country of birth } \\
\hline Australia & $95(66.9)$ \\
\hline Other specified country & $46(32.4)$ \\
\hline Unspecified & $1(0.7)$ \\
\hline \multicolumn{2}{|l|}{ Primary language spoken at home } \\
\hline English & $101(71.1)$ \\
\hline Other specified language & $41(28.9)$ \\
\hline \multicolumn{2}{|l|}{ Household income (Aus \$) ${ }^{a}$} \\
\hline Less than $\$ 50,000$ per year & $38(26.8)$ \\
\hline$\$ 50,001-\$ 100,000$ per year & $33(23.2)$ \\
\hline More than $\$ 100,000$ per year & $52(36.6)$ \\
\hline Unsure or unspecified & $19(13.4)$ \\
\hline
\end{tabular}

${ }^{\mathrm{a} A u s} \$ 1=\mathrm{US} \$ 0.70$.

Table 2. Representativeness of responding participants.

\begin{tabular}{lll}
\hline Characteristic & Graduates $(\mathrm{N}=8532), \mathrm{n}(\%)$ & Respondents $(\mathrm{n}=142), \mathrm{n}(\%)^{P \text { value }^{\mathrm{a}}}$ \\
\hline $\begin{array}{l}\text { Gender }^{\mathbf{b}} \\
\text { Female }\end{array}$ & $4758(55.8)$ & $100(70.9)$ \\
$\quad$ Male & $3774(44.2)$ & $41(29.1)$ \\
Level of award & & \\
$\quad$ Undergraduate & $5480(64.2)$ & $82(58.2)$ \\
$\quad$ Postgraduate & $3052(35.8)$ & $59(41.8)$ \\
Graduation time & & .001 \\
$\quad$ April & $5308(62.2)$ & $78(54.9)$ \\
$\quad$ September & $3224(37.8)$ & $64(45.1)$ \\
\hline
\end{tabular}

${ }^{\mathrm{a}} \mathrm{Chi}$-square test for difference in proportions.

${ }^{\mathrm{b}}$ Gender was missing for $\mathrm{n}=1$ responding participant, who was omitted from the chi-square test.

${ }^{c}$ Level of award was missing for $\mathrm{n}=1$ responding participant, who was omitted from the chi-square test.

Of the 142 participants who answered the baseline questionnaire, 99 also completed the 12-month follow-up survey. This equated to a retention rate of $69.7 \%$. The completeness of the baseline and 12-month follow-up surveys is shown in Table 3.
Of the 238 graduates who registered to participate in this study, 161 consented to having their survey responses linked to their health records, while 77 opted out of the data linkage component of this study. Thus, the data linkage opt-out rate was $32.4 \%$. Of 
the 161 registered participants who consented having their survey responses linked to their health records, 46 had used health services and were linked to health records in the CHeReL Master Linkage Key. This equated to a data linkage rate of 28.6\%. The most commonly available linked health records were hospital episodes of care and emergency department presentations (Table 4). Unsurprisingly, there were no linked mortality data for this study period. Linkage with cancer registry records was not possible because the most recent cancer registry update preceded the follow-up period for the present study.

Table 3. Completeness of survey components at baseline and 12-month follow-up. Completeness was calculated as the number of survey items within a specific survey domain that had missing data, divided by the cross product of the total number of survey items within the specific survey domain and the number of participants not lost to follow-up when the survey was administered.

\begin{tabular}{|c|c|c|}
\hline Domain/Instrument & Baseline (\%) & Follow-up (\%) \\
\hline \multicolumn{3}{|l|}{ Sociodemographic factors } \\
\hline Questions about personal status & 99.8 & 99.7 \\
\hline Questions about tertiary education & 98.8 & 100.0 \\
\hline Questions about employment status & 97.9 & 99.0 \\
\hline \multicolumn{3}{|l|}{ Working life } \\
\hline Questions about job satisfaction & 97.5 & 96.5 \\
\hline Questions about career mentoring & 97.3 & 98.0 \\
\hline Role Balance Scale (RBS) & 97.1 & 98.0 \\
\hline \multicolumn{3}{|l|}{ Health and lifestyle } \\
\hline Questions about physical activity & 96.4 & 96.8 \\
\hline Questions about health risk factors & 91.5 & 94.6 \\
\hline Short Form Health Survey (SF-12) & 95.6 & 95.8 \\
\hline EuroQoL 5-dimension (EQ-5D) & 94.8 & 95.8 \\
\hline General Anxiety Disorder scale (GAD-7) & 94.5 & 95.1 \\
\hline Social Interaction Anxiety Scale (SIAS-6) & 95.1 & 94.6 \\
\hline Kessler Psychological Distress Scale (K10) & 94.3 & 94.6 \\
\hline Questions about injury & 92.3 & 94.9 \\
\hline \multicolumn{3}{|l|}{ Social support and resilience } \\
\hline Questions about social connectedness & 93.0 & 94.9 \\
\hline Brief Resilience Scale (BRS) & 92.8 & 94.9 \\
\hline Multidimensional Scale of Perceived Social Support (MSPSS) & 92.2 & 94.8 \\
\hline Social Readjustment Rating Scale (SRRS) & 89.4 & 93.9 \\
\hline \multicolumn{3}{|l|}{ Caregiver activities } \\
\hline Questions about caregiver responsibilities and activities & 89.4 & 92.9 \\
\hline \multicolumn{3}{|l|}{ Social media and technology } \\
\hline Questions about use of social networking sites & 89.1 & 89.5 \\
\hline Questions about social media experiences & 87.3 & 89.9 \\
\hline
\end{tabular}


Table 4. Availability of linked records.

\begin{tabular}{ll}
\hline Data source & Respondents $(\mathrm{n}=46), \mathrm{n}(\%)$ \\
\hline NSW ${ }^{\mathrm{a}}$ Emergency Department Data Collection & $37(80)$ \\
NSW Admitted Patient Data Collection & $31(67)$ \\
NSW Ambulance - electronic medical record & $6(13)$ \\
NSW Registry of Births, Deaths and Marriages - Death registrations & $0(0)$ \\
NSW Cause of Death - Unit Record File & $0(0)$ \\
NSW Central Cancer Registry & N/A \\
\hline
\end{tabular}

${ }^{a}$ NSW: New South Wales.

${ }^{b}$ Linkage not possible because most recent records preceded the follow-up period for this study.

${ }^{\mathrm{c}}$ N/A: not applicable. At the time of linkage, the NSW Central Cancer Registry data for the study period were not yet available in the Master Linkage Key.

\section{Discussion}

This pilot study examined the feasibility of recruiting university graduates to establish a longitudinal cohort study to inform our understanding of emerging adulthood. It found that the overall recruitment rate was poor, while the completeness of survey responses among respondents was good to excellent. There was moderate acceptability for conducting data linkage of health records from administrative data collections and survey responses.

\section{Survey Recruitment and Response Rates}

Of the $2.8 \%$ (238/8532) of invited graduates who agreed to participate in this pilot cohort study, 59.7\% (142/238) responded to the baseline survey. This is considerably lower than the response rate for, for instance, the pilot SUN study (11\%) [13] and the Australian arm of the World Health Organization's World Mental Health Surveys International College Student initiative (7\%) [14]. Although the overall recruitment and response rates are disappointing, they are perhaps unsurprising given the general decline in survey participation observed in recent decades [15-17]. Superimposed on this general decline, there is a myriad of factors that may have contributed to the relatively poor recruitment and response rates observed in this pilot cohort study. Dillman's extension of social exchange theory, the tailored design method, is a theoretical framework that seeks to explain why individuals are motivated to engage in certain social behaviors such as survey participation [18]. This framework suggests that survey response rates depend on reward, cost, and trust. For instance, survey participation is typically more rewarding when participants have a vested interest in the topic [19]. The time spent completing a survey is an important cost consideration for survey participants [19], with longer stated survey length resulting in fewer respondents [20]. In addition to survey length, poor survey structure and design can increase the perceived cost of responding to surveys [19]. In regard to trust, perceived trustworthiness of the organization or institution responsible for administering the survey, confidential use of data, and adequate privacy protections are key elements for reassuring survey participants and improving response rates [19].
It is difficult to determine to which extent each of the abovementioned factors have influenced the recruitment and response rates in this pilot cohort study. Although one might expect recent university graduates to have a vested interest in the topic of health and well-being in emerging adults, perhaps the relative absence of health problems in this age group resulted in a lower interest in the topic and thus lower perceived reward and motivation for participation. For instance, there is evidence suggesting that emerging adults are less motivated by long-term health concerns and lifestyle interventions than older counterparts [21-24]. Additionally, perhaps graduating from university is accompanied by a sense of separation and decreased interest in participating in university-based research surveys. There is also the potential issue of different surveys competing for graduates' attention and motivation. For instance, Australian university graduates are regularly invited to complete the nationwide Graduate Outcomes Survey, which is one component of the Quality Indicators for Learning and Teaching suite of surveys conducted for the Australian Government Department of Education, Skills and Employment [25]. Competition for attention and motivation becomes a particularly important consideration in the context of emerging adults' perceived scarcity of time [26]. The graduates invited to participate in this pilot study were informed that it would take approximately 40 minutes to complete each survey (ie, baseline and follow-up). It is conceivable that many potential participants considered the perceived costs of participation in this study to be too high.

Although there is conflicting evidence, some studies have demonstrated that incentives such monetary rewards or lotteries can positively impact response rates $[27,28]$. In an attempt to improve the recruitment rate in this pilot study, an incentive to participate was introduced for the second (ie, September) graduation period. Consequently, the recruitment rate increased from $2 \%$ for the first (ie, April) graduation cohort to $3.8 \%$ for the second graduation cohort. The improvement after introducing incentives notwithstanding, the recruitment rate remained disappointingly low. This suggests that the incentives to participate in this pilot study were insufficient to counteract the perceived costs of participation for the vast majority of invited graduates. Perhaps the proliferation and ubiquity of mobile devices in modern society means that the main incentive 
used in this pilot study (ie, a chance to win an iPad mini) is not perceived as an attractive reward for participating in research.

Previous research has demonstrated that multiple reminders are an effective way to increase response rates [29-31]. However, studies have also shown that the recruitment yield typically declines rapidly with each subsequent reminder [32-34]. This rapidly diminishing marginal return suggests that increasing the number of reminders beyond a small number may not be a cost-effective measure [35]. Hence, Saleh and Bista [19] recommended sending at least 1 reminder, but not more than 3 . Furthermore, the desire for increased survey response rates also needs to be balanced with the concern among human research ethics committees that multiple reminders may result in potential survey participants feeling harassed or coerced into participating in the research $[36,37]$.

\section{Survey Completeness}

Survey completeness can be used to refer to 3 different concepts: (1) completeness of the achieved sample with respect to the original one, (2) participation of the respondents throughout all the phases predicted by a research design, and (3) respondents' propensity to answer all the questions within the questionnaire [29]. The latter 2 concepts were considered in this pilot study. The first of which corresponds to the retention rate between the baseline and follow-up surveys, which was found to be $69.7 \%$ (99/142). In regard to the propensity to answer all the questions within a survey, the completeness was excellent, with the proportion of questions answered in each survey domain ranging from $87 \%$ to $100 \%$ in both the baseline and follow-up surveys. This suggests that the participants did not consider the survey length to be excessive in the pilot study. This is important because previous research has shown that longer survey length can result in both poorer completeness and quality of responses [26].

\section{Data Linkage}

Approximately two-thirds (161/238,67.6\%) of the participants in this pilot study did not opt out of having their survey responses linked to their health records. This suggests that the acceptability of data linkage in the present study was very similar to that in previous Australian studies. For instance, one study reported that $66 \%$ of older Australians found it acceptable to have their health data accessed and linked in a registry [38]. Another study of young Australian parents reported, unsurprisingly, that privacy protection was an important consideration for most participants [39]. However, it also was noted that protection measures adopted in best practice health data linkage studies were viewed by most participants as adequate protection for data linkage to proceed without specific individual consent.

\section{Recommendations}

This pilot study offers insights into the feasibility of recruiting recent university graduates to establish a longitudinal cohort study to investigate health and well-being in emerging adults. It is unrealistic to expect a reversal of the general decline in survey response rates. However, it has been suggested that reducing nonresponse rates is less important than minimizing bias in estimates. That is, despite preconceived notions of a good response rate, neither a $5 \%$ response rate nor even a $75 \%$ response rate necessarily provides unbiased estimates [40,41]. Nonresponse bias occurs when subgroups respond at different rates. In this pilot study, female graduates were more likely to respond to the baseline survey. Overrepresentation of females is common in health-related survey research. Although postsurvey adjustment techniques can be implemented to help reduce nonresponse biases, it is often preferable to prevent nonresponse bias by designing surveys that are more acceptable to the target population in the first instance [42].

In the context of this pilot study, future cohort studies of emerging adults should target populations that are more likely to hold interest in the research. For instance, it might be more useful to target populations at an earlier stage, such as at first enrollment at university or before leaving high school. There are potentially more opportunities for targeted engagement with a student cohort who are commencing postsecondary education. Only a subset of emerging adults undertakes postsecondary education. In Australia, 39.7\% of people aged 25 to 34 years had a bachelor's degree or higher in 2018 [43]. Thus, a cohort of high school graduates offers a less biased sample.

Apart from the choice of specific target populations, future studies should consider the need for personalizing invitations, reducing survey length, crafting surveys that are simple to complete, administering surveys via smartphone apps, and incentivizing participation (eg, through gamification). Lastly, future studies are strongly encouraged to use research codesign to optimize survey parameters. Incorporating the lived experiences of emerging adults into the development and implementation of future research will increase its likelihood of success and impact $[44,45]$.

\section{Conclusions}

The overall recruitment rate was poor, while the completeness of survey responses among respondents was good to excellent. There was reasonable acceptability for conducting data linkage of health records from administrative data collections and survey responses. Future research aiming to establish a longitudinal cohort study to investigate health and well-being in emerging adults should carefully consider the target population as well as how best to obtain an unbiased sample and craft surveys to maximize participation.

\section{Acknowledgments}

The authors wish to thank the Macquarie University Graduation Office for assisting with participant recruitment and supplying basic information about the 2018 graduate cohort. The authors wish to thank the New South Wales (NSW) Ministry of Health for providing access to their Admitted Patient Data Collection; Emergency Department Data Collection; Registry of Births, Deaths and Marriages - Death registrations; and Cause of Death Unit Record File. The authors also wish to thank the NSW Ambulance for providing access to their electronic medical record data collection, Cancer Institute NSW for providing access to their NSW 
Central Cancer Registry, and the CHeReL for conducting the secure data linkages. This study was funded by a Macquarie University Research Seeding Grant (grant reference number: 38278336). The funding body had no role in the design of this study, data analysis, interpretation of findings, or preparation of manuscripts. Ethical approval for this pilot longitudinal cohort study was granted by the Macquarie University Human Research Ethics Committee (5201600265) and the NSW Population and Health Service Research Ethics Committee (2020/ETH01748/2019.57).

\section{Authors' Contributions}

All authors contributed to the conception and design of the study. DFP and RPL were responsible for drafting the first version of the manuscript. JA, JB, CMC, RJM, and VW were responsible for supervising the project. All authors contributed to or edited the manuscript. All authors read and approved the final version of the manuscript.

\section{Conflicts of Interest}

None declared.

\section{Multimedia Appendix 1}

Multiple regression analysis for socioeconomic and clinical factors affecting anxiety and depressive disorders in the local community group.

[DOCX File, 16 KB-Multimedia Appendix 1]

\section{Multimedia Appendix 2}

Multiple regression analysis for socioeconomic and clinical factors affecting anxiety and depressive disorders in the foreign community group.

[DOCX File, 16 KB-Multimedia Appendix 2]

\section{References}

1. Arnett JJ. Emerging adulthood. A theory of development from the late teens through the twenties. Am Psychol 2000;55(5):469-480. [Medline: 10842426]

2. Arnett J. Emerging Adulthood: The Winding Road from the Late Teens Through the Twenties, 2nd Edition. New York, NY: Oxford University Press; 2014.

3. Buhl HM. Well-being and the child-parent relationship at the transition from university to work life. J Adolesc Res 2007;22(5):550-571. [doi: 10.1177/0743558407305415]

4. Tanner J. Mental health in emerging adulthood. In: Arnett JJ, editor. The Oxford Handbook of Emerging Adulthood. New York, NY: Oxford University Press; 2015.

5. Barlett CP, Barlett ND. The young and the restless: examining the relationships between age, emerging adulthood variables, and the Dark Triad. Pers Individ Dif 2015;86:20-24. [doi: 10.1016/j.paid.2015.05.024]

6. Stampfer MJ, Hu FB, Manson JE, Rimm EB, Willett WC. Primary prevention of coronary heart disease in women through diet and lifestyle. N Engl J Med 2000;343(1):16-22. [doi: 10.1056/NEJM200007063430103] [Medline: 10882764]

7. Cao Y, Willett WC, Rimm EB, Stampfer MJ, Giovannucci EL. Light to moderate intake of alcohol, drinking patterns, and risk of cancer: results from two prospective US cohort studies. BMJ 2015;351:h4238. [doi: 10.1136/bmj.h4238] [Medline: 26286216]

8. Martínez-González MA. The SUN cohort study (Seguimiento University of Navarra). Public Health Nutr 2006;9(1A):127-131. [doi: 10.1079/phn2005935] [Medline: 16512960]

9. Seguí-Gómez M, de la Fuente C, Vázquez Z, de Irala J, Martínez-González MA. Cohort profile: the 'Seguimiento Universidad de Navarra' (SUN) study. Int J Epidemiol 2006;35(6):1417-1422. [doi: 10.1093/ije/dyl223] [Medline: 17060332]

10. Eisenberg ME, Spry E, Patton GC. From emerging to established: longitudinal patterns in the timing of transition events among Australian emerging adults. Emerg Adulthood 2015;3(4):277-281. [doi: 10.1177/2167696815574639]

11. Landstedt E, Coffey J, Nygren M. Mental health in young Australians: a longitudinal study. J Youth Stud 2015;19(1):74-86. [doi: 10.1080/13676261.2015.1048205]

12. Lystad RP, Fajardo Pulido D, Peters L, Johnstone M, Ellis LA, Braithwaite J, et al. Monitoring health and well-being in emerging adults: protocol for a pilot longitudinal cohort study. JMIR Res Protoc 2020;9(4):e16108 [FREE Full text] [doi: 10.2196/16108] [Medline: 32324142]

13. Sánchez-Villegas A, de IJ, Martínez-González M. Dieta Mediterránea y enfermedad cardiovascular: Resultados del estudio piloto del proyecto SUN. Rev Med Univ Navarra 2002;46(3):9-16 [FREE Full text] [doi: 10.1016/s0025-7753(02)73311-3]

14. Auerbach RP, Mortier P, Bruffaerts R, Alonso J, Benjet C, Cuijpers P, WHO WMH-ICS Collaborators. Mental disorder comorbidity and suicidal thoughts and behaviors in the World Health Organization World Mental Health Surveys International College Student initiative. Int J Methods Psychiatr Res 2019;28(2):e1752 [FREE Full text] [doi: 10.1002/mpr.1752] [Medline: $\underline{30450753}$ ] 
15. Brick JM, Williams D. Explaining rising nonresponse rates in cross-sectional surveys. Ann Am Acad Pol Soc Sci 2012;645(1):36-59. [doi: 10.1177/0002716212456834]

16. Meyer BD, Mok WKC, Sullivan JX. Household surveys in crisis. J Econ Perspect 2015;29(4):199-226. [doi: 10.1257/jep.29.4.199]

17. Williams D, Brick J. Trends in U.S. face-to-face household survey nonresponse and level of effort. J Surv Stat Methodol 2018;6(2):186-211. [doi: 10.1093/jssam/smx019]

18. Dillman DA, Smyth JD, Christian LM. Internet, Phone, Mail, and Mixed-Mode Surveys: The Tailored Design Method, 4th Edition. Hoboken, NJ: Wiley; 2014.

19. Saleh A, Bista K. Examining factors impacting online survey response rates in educational research: perceptions of graduate students. J Multidiscip Eval 2017;13(29):63-74.

20. Galesic M, Bosnjak M. Effects of questionnaire length on participation and indicators of response quality in a web survey. Public Opin Q 2009;73(2):349-360. [doi: 10.1093/poq/nfp031]

21. Lanoye A, Grenga A, Leahey TM, LaRose JG. Motivation for weight loss and association with outcomes in a lifestyle intervention: comparing emerging adults to middle aged adults. Obes Sci Pract 2019;5(1):15-20 [FREE Full text] [doi: 10.1002/osp4.313] [Medline: 30847223]

22. LaRose JG, Leahey TM, Hill JO, Wing RR. Differences in motivations and weight loss behaviors in young adults and older adults in the National Weight Control Registry. Obesity 2013;21(3):449-453 [FREE Full text] [doi: 10.1002/oby.20053] [Medline: 23404944]

23. LaRose JG, Guthrie KM, Lanoye A, Tate DF, Robichaud E, Caccavale LJ, et al. A mixed methods approach to improving recruitment and engagement of emerging adults in behavioural weight loss programs. Obes Sci Pract 2016;2(4):341-354 [FREE Full text] [doi: 10.1002/osp4.71] [Medline: 28090339]

24. Corsino L, Lin P, Batch BC, Intille S, Grambow SC, Bosworth HB, et al. Recruiting young adults into a weight loss trial: report of protocol development and recruitment results. Contemp Clin Trials 2013;35(2):1-7. [doi: 10.1016/j.cct.2013.04.002] [Medline: 23591327]

25. Graduate Outcomes Survey. Quality Indicators for Learning and Teaching. URL: https://www.qilt.edu.au/surveys/ graduate-outcomes-survey-(gos)

26. Edwards PJ, Roberts I, Clarke MJ, Diguiseppi C, Wentz R, Kwan I, et al. Methods to increase response to postal and electronic questionnaires. Cochrane Database Syst Rev 2009(3):MR000008. [doi: 10.1002/14651858.MR000008.pub4] [Medline: 19588449]

27. Heerwegh D. An investigation of the effect of lotteries on web survey response rates. Field Methods 2016;18(2):205-220. [doi: 10.1177/1525822X05285781]

28. Laguilles JS, Williams EA, Saunders DB. Can lottery incentives boost web survey response rates? Findings from four experiments. Res High Educ 2010;52(5):537-553. [doi: 10.1007/s11162-010-9203-2]

29. Christensen AI, Ekholm O, Kristensen PL, Larsen FB, Vinding AL, Glümer C, et al. The effect of multiple reminders on response patterns in a Danish health survey. Eur J Public Health 2015;25(1):156-161. [doi: 10.1093/eurpub/cku057] [Medline: 24855288]

30. Strazdins L, Griffin AL, Broom DH, Banwell C, Korda R, Dixon J, et al. Time scarcity: another health inequality? Environ Plan A 2011;43(3):545-559. [doi: 10.1068/a4360]

31. Decataldo A, Denti F, Amico A. Increasing participation rates and data quality in e-mail survey. An experimental design of research. Sociol Ric Soc 2020;122:45-68. [doi: 10.3280/sr2020-122003]

32. Brøgger J, Bakke P, Eide GE, Gulsvik A. Contribution of follow-up of nonresponders to prevalence and risk estimates: a Norwegian respiratory health survey. Am J Epidemiol 2003;157(6):558-566. [doi: 10.1093/aje/kwg003] [Medline: 12631547]

33. Elliott AM, Hannaford PC. Third mailings in epidemiological studies: are they really necessary? Fam Pract 2003;20(5):592-594. [doi: 10.1093/fampra/cmg517] [Medline: 14507804]

34. Uusküla A, Kals M, McNutt L. Assessing non-response to a mailed health survey including self-collection of biological material. Eur J Public Health 2011;21(4):538-542. [doi: 10.1093/eurpub/ckq053] [Medline: 20457781]

35. Breen CL, Shakeshaft AP, Doran CM, Sanson-Fisher RW, Mattick RP. Cost-effectiveness of follow-up contact for a postal survey: a randomised controlled trial. Aust N Z J Public Health 2010;34(5):508-512. [doi: 10.1111/j.1753-6405.2010.00598.x] [Medline: 21040180]

36. Schirmer J. Ethical issues in the use of multiple survey reminders. J Acad Ethics 2009;7:125-139. [doi: $10.1007 / \mathrm{s} 10805-009-9072-5]$

37. Howell SC, Quine S, Talley NJ. Ethics review and use of reminder letters in postal surveys: are current practices compromising an evidence-based approach? Med J Aust 2003;178(1):43. [doi: 10.5694/j.1326-5377.2003.tb05043.x] [Medline: 12492391]

38. Harrison SL, Milte R, Bradley C, Inacio M, Crotty M. The acceptability of participating in data linkage research: research with older Australians. Aust N Z J Public Health 2018;42(5):497-498. [doi: 10.1111/1753-6405.12797] [Medline: 29896924]

39. Xafis V. The acceptability of conducting data linkage research without obtaining consent: lay people's views and justifications. BMC Med Ethics 2015;16(1):79 [FREE Full text] [doi: 10.1186/s12910-015-0070-4] [Medline: 26577591] 
40. Fosnacht K, Sarraf S, Howe E, Peck LK. How important are high response rates for college surveys? Rev Hig Educ 2017;40(2):245-265. [doi: 10.1353/rhe.2017.0003]

41. Hendra R, Hill A. Rethinking response rates: new evidence of little relationship between survey response rates and nonresponse bias. Eval Rev 2019;43(5):307-330. [doi: 10.1177/0193841X18807719] [Medline: 30580577]

42. Toepoel V, Schonlau M. Dealing with nonresponse: strategies to increase participation and methods for postsurvey adjustments. Math Popul Stud 2017;24(2):79-83. [doi: 10.1080/08898480.2017.1299988]

43. 6227.0 - Education and work, Australia, May 2018. Australian Bureau of Statistics. Canberra, Australia; 2018. URL: https:/ /www.abs.gov.au/AUSSTATS/abs@.nsf/allprimarymainfeatures/D6172962B0F8799FCA2584B0000EEBC3?opendocument [accessed 2021-10-12]

44. Slattery P, Saeri AK, Bragge P. Research co-design in health: a rapid overview of reviews. Health Res Policy Syst 2020;18(1):17 [FREE Full text] [doi: 10.1186/s12961-020-0528-9] [Medline: 32046728]

45. Rouncefield-Swales A, Harris J, Carter B, Bray L, Bewley T, Martin R. Children and young people's contributions to public involvement and engagement activities in health-related research: a scoping review. PLoS One 2021;16(6):e0252774 [FREE Full text] [doi: 10.1371/journal.pone.0252774] [Medline: $\underline{\text { 34106978] }}$

\author{
Abbreviations \\ ACTRN: Australian New Zealand Clinical Trials Registry \\ CHeReL: Centre for Health Record Linkage \\ HRQoL: health-related quality of life \\ NSW: New South Wales \\ SUN: Seguimiento Universidad de Navarra
}

\author{
Edited by G Eysenbach; submitted 03.05.21; peer-reviewed by T Lefèvre, D Micallef; comments to author 06.10.21; revised version \\ received 13.10.21; accepted 17.11.21; published 06.01.22 \\ Please cite as: \\ Lystad RP, Fajardo Pulido D, Peters L, Johnstone M, Ellis LA, Braithwaite J, Wuthrich V, Amin J, Cameron CM, Mitchell RJ \\ Feasibility of Monitoring Health and Well-being in Emerging Adults: Pilot Longitudinal Cohort Study \\ JMIR Form Res 2022;6(1):e30027 \\ URL: https://formative.jmir.org/2022/1/e30027 \\ doi: $10.2196 / 30027$ \\ PMID: 34989696
}

CReidar P Lystad, Diana Fajardo Pulido, Lorna Peters, Melissa Johnstone, Louise A Ellis, Jeffrey Braithwaite, Viviana Wuthrich, Janaki Amin, Cate M Cameron, Rebecca J Mitchell. Originally published in JMIR Formative Research (https://formative.jmir.org), 06.01.2022. This is an open-access article distributed under the terms of the Creative Commons Attribution License (https://creativecommons.org/licenses/by/4.0/), which permits unrestricted use, distribution, and reproduction in any medium, provided the original work, first published in JMIR Formative Research, is properly cited. The complete bibliographic information, a link to the original publication on https://formative.jmir.org, as well as this copyright and license information must be included. 